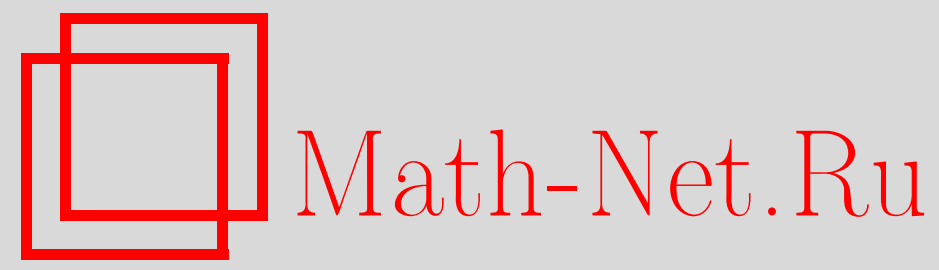

В. Ф. Гапошкин, Спектральные критерии существования обобщенных эргодических преобразований, Теория вероятн. и ее примен., 1996, том 41, выпуск 2, 251-271

DOI: https://doi.org/10.4213/tvp2931

Использование Общероссийского математического портала Math-Net.Ru подразумевает, что вы прочитали и согласны с пользовательским соглашением http://www . mathnet.ru/rus/agreement

Параметры загрузки:

IP: 44.207 .124 .84

26 апреля 2023 г., $16: 45: 24$

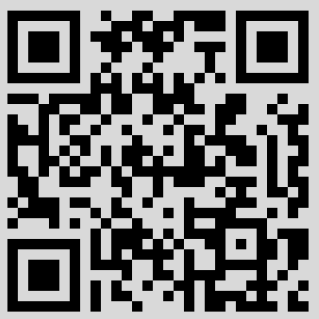


(C) 1996 r.

ГАПОШКИН В. $\Phi . *$

\title{
СПЕКТРАЛЬНЫЕ КРИТЕРИИ СУЩЕСТВОВАНИЯ ОБОБЩЕННЫХ ЭРГОДИЧЕСКИХ ПРЕОБРАЗОВАНИЙ
}

\begin{abstract}
Изучаются условия сходимости почти всюду односторонних и двусторонних эргодических преобразований

$$
\sum_{1}^{\infty} b_{k} T^{k} f(x) \quad \text { и } \sum_{-\infty}^{\infty} b_{k} T^{k} f(x)
$$

где $T-$ унитарный оператор в $L^{2}$. Получены критерии существования этих преобразований, выраженные через свойства спектральной меры операторов. Рассматриваются аналоги этих результатов для нормальных операторов, стационарных и гармонизуемых случайных процессов.
\end{abstract}

Ключевые слова и фразы: унитарные операторы, нормальные операторы, стационарные процессы, гармонизуемые процессы, спектральная мера, эргодические преобразования, сходимость почти всюду.

\section{Введение}

Если $(\Omega, \mathcal{A}, \mu)$ - некоторое измеримое пространство, $T$ - унитарный оператор в $L^{2}(\Omega)$, то достаточно детально изучен вопрос о сходимости в $L^{2}$ и почти всгопу средних

$$
n^{-1} \sum_{k=1}^{n} T^{k} f(x)
$$

или средних $(2 n+1)^{-1} \sum_{k=-n}^{n} T^{k} f(x)$ при $n \rightarrow \infty$. В вероятностных терминах эта задача формулируется как усиленный закон больших чисел для стационарных в широком смысле процессов $\left(\xi_{k}\right)$.

Необходимые и достаточные условия сходимости п.в. средних (1) получены в работах автора [1], [2] в терминах спектральной меры оператора $T$. Пусть $d U(\lambda)$ - операторная мера с ортогональными приращениями, для $f \in L^{2}$ обозначим $d Z(\lambda)=d U(\lambda) f ;\|d Z(\lambda)\|^{2}=d F(\lambda)$.

*Московский государственный университет путей сообщения, кафедра прикладной математики, ул. Образцова, 15, 103055 Москва, Россия.

1) Работа поддержана внебюджетным фондом НИР МПС РФ и РФФИ, грант № 96-01-01129. 
Тогда

$$
T^{k} f=\int_{-\pi}^{\pi} e^{i k \lambda} d Z(\lambda) ; \quad \int_{-\pi}^{\pi} d F(\lambda)=\|f\|^{2}<\infty .
$$

Как известно, средние (1) для любой фиксированной функции $f \in L^{2}$ сходятся в $L^{2}$ к значению $\xi=F(+0)-F(-0)$. В работах [1], [2] показано, что для сходимости этих средних п.в. необходимо и достаточно, чтобы

$$
\lim _{m \rightarrow \infty} \int_{0<|\lambda| \leqslant 2^{-m}} d Z(\lambda)=0 \quad \text { п.в. }
$$

В частности, это условие выполнено, если

$$
\int_{0<|\lambda| \leqslant \pi} L_{2}^{2}(\lambda) d Z(\lambda)<\infty .
$$

Здесь и далее используются обозначения

$$
L_{1}(\lambda)=\log \frac{2 \pi}{|\lambda|} ; \quad L_{2}(\lambda)=\log \log \frac{4 \pi}{|\lambda|}, \quad L_{3}(\lambda)=\log \log \log \frac{16 \pi}{|\lambda|}, \ldots
$$

(все логарифмы берутся по основанию 2). Наряду со средними (1) в эргодической теории изучалось также (двустороннее) эргодическое преобразование Гильберта последовательности $\left\{T^{k} f(x)\right\}$ :

$$
\sigma_{n}^{\prime}=\sum_{k=-n}^{n}{ }^{\prime} k^{-1} T^{k} f(x)=\sum_{k=1}^{n}{ }^{\prime} k^{-1}\left(T^{k} f(x)-T^{-k} f(x)\right) .
$$

Известно, что $\lim _{n \rightarrow \infty} \sigma_{n}^{\prime}=\eta$ в $L^{2}$ всегда существует. В работе [3] тем же методом, что и в [1], [2], показано, что эргодическое преобразование Гильберта (5) сходится п.в. тогда и только тогда, когда

$$
\lim _{m \rightarrow \infty} \int_{0<|\lambda| \leqslant 2^{-m}} \operatorname{sign} \lambda d Z(\lambda)=0 \quad \text { п.в. }
$$

3 а м е ч а н и е. Последний результат тесно связан с критерием сходимости п.в. разложения Котельникова-ШІеннона для стационарного процесса с ограниченным спектром [2]. Если спектр процесса $\tilde{\xi}(t)$ сосредоточен на $[-\pi, \pi]$, это разложение имеет вид

$$
\tilde{\xi}(t)=\int_{-\pi}^{\pi} e^{i \lambda t} d \tilde{Z}(\lambda)=\lim _{n \rightarrow \infty} \sum_{k=-n}^{n} \tilde{\xi}(k) \frac{\sin \pi(t-k)}{\pi(t-k)} .
$$

Ряд (7) сходится п.в. (равномерно на любом конечном интервале $|t| \leqslant T$ ) тогда и только тогда, когда

$$
\lim _{m \rightarrow \infty}\left[\int_{0<|\lambda+\pi| \leqslant 2^{-m}} d \tilde{Z}(\lambda)-\int_{0<|\lambda-\pi| \leqslant 2^{-m}} d \widetilde{Z}(\lambda)\right]=0 \quad \text { п.в. }
$$


Очевидно, что сходимость п.в. ряда (7) эквивалентна существованию п.в. предела $\lim _{n \rightarrow \infty} \sum_{k=-n}^{n}(-1)^{k} k^{-1} \xi(k)$, т.е. существованию эргодического преобразования Гильберта для стационарной последовательности $\xi_{k}=(-1)^{k} \tilde{\xi}_{k}$. Условие (6) для последовательности $\left(\xi_{k}\right)$ эквивалентно условию $\left(6^{\prime}\right)$.

В работах [4]-[10] рассматривались аналоги и обобщения этих результатов для других классов операторов, случайных процессов и полей.

В § 1 данной статьи исследуется сходимость п.в. одностороннего эргодического преобразования Гильберта, т.е. средних

$$
\sigma_{n}^{\prime \prime}=\sum_{k=1}^{n} k^{-1} T^{k} f(x), \quad \sigma_{n}=\sum_{k=1}^{n} k^{-1}\left(T^{k} f(x)+T^{-k} f(x)\right) .
$$

В 2 рассматриваются обобщенные двусторонние и односторонние эргодические преобразования, в которых вместо коэффициентов $k^{-1}$ берутся коэффициенты $k^{-\alpha}, 0<\alpha<1$. В 33 изучаются некоторые другие классы операторов и случайных процессов, а также более обшие коэффициенты преобразований.

\section{§1. Одностороннее преобразование Гильберта}

В этом параграфе $T$ - унитарный оператор в $L^{2}, f \in L^{2}$. Тогда имеет место представление (2). Рассмотрим средние $\sigma_{n}, \sigma_{n}^{\prime}, \sigma_{n}^{\prime \prime}$ :

$$
\begin{aligned}
\sigma_{n}^{\prime} & =\sum_{k=1}^{n} k^{-1}\left(T^{k} f(x)-T^{-k} f(x)\right)=2 i \int_{|\lambda| \leqslant \pi} Q_{n}^{\prime}(\lambda) d Z(\lambda), \\
\sigma_{n} & =\sum_{k=1}^{n} k^{-1}\left(T^{k} f(x)+T^{-k} f(x)\right)=2 \int_{|\lambda| \leqslant \pi} Q_{n}(\lambda) d Z(\lambda), \\
\sigma_{n}^{\prime \prime} & =\sum_{k=1}^{n} k^{-1} T^{k} f(x)=\int_{|\lambda| \leqslant \pi} Q_{n}^{\prime \prime}(\lambda) d Z(\lambda),
\end{aligned}
$$

где

$$
\begin{aligned}
& Q_{n}^{\prime}(\lambda)=\sum_{k=1}^{n} k^{-1} \sin k \lambda, \quad Q_{n}(\lambda)=\sum_{k=1}^{n} k^{-1} \cos k \lambda \\
& Q_{n}^{\prime \prime}(\lambda)=Q_{n}(\lambda)+i Q_{n}^{\prime}(\lambda) .
\end{aligned}
$$

Известно, что $Q_{n}^{\prime}(\lambda)=O(1)$ равномерно по $\lambda(n \rightarrow \infty)$;

$$
\lim _{n \rightarrow \infty} Q_{n}(\lambda)=\Phi(\lambda) ; \quad \Phi(\lambda) \sim \log \frac{2 \pi}{|\lambda|} \quad \text { при } \quad \lambda \rightarrow 0 .
$$

Отсюда сразу следует

Теорема 1. Средние $\sigma_{n}\left(\sigma_{n}^{\prime \prime}\right)$ сходятся в $L^{2} \kappa \eta\left(\eta^{\prime \prime}\right)$ mогда и только mozda, когдa

$$
F(+0)=F(-0)
$$


и выполнено условие

$$
\int_{0<|\lambda| \leqslant \pi} L_{1}^{2}(\lambda) d F(\lambda)<\infty
$$

При этом

$$
\begin{aligned}
& \eta \stackrel{L^{2}}{=} 2 \int_{0<|\lambda| \leqslant \pi} \Phi(\lambda) d Z(\lambda), \\
& \eta^{\prime \prime} \stackrel{L^{2}}{=} \int_{0<|\lambda| \leqslant \pi} \Phi(\lambda) d Z(\lambda)+i \int_{0<|\lambda| \leqslant \pi} \frac{\pi-|\lambda|}{2} \operatorname{sign} \lambda d Z(\lambda) .
\end{aligned}
$$

Далее нас будут интересовать условия сходимости средних $\sigma_{n}, \sigma_{n}^{\prime \prime}$ не только в $L^{2}$, но и почти вскду. Так как условие (9) влечет за собой условие $\left(3^{\prime}\right)$ и сходимость п.в. средних $\sigma_{n}^{\prime}$, можно ограничиться исследованием лишь средних $\sigma_{n}$. Условия сходимости н.в. средних $\sigma_{n}^{\prime \prime}$ аналогичны.

Теорема 2. Пусть выполнены условия (8)-(10). Обозначим $N_{m}=2^{2^{m}}, \lambda_{m}=2 \pi / N_{m}$.

1) Справедливо следующее представление средних $\sigma_{\nu}$ : если $N_{m} \leqslant$ $\nu<N_{m+1}$, mo

$$
\sigma_{\nu}=\eta-2 \int_{0<|\lambda| \leqslant \lambda_{m}} L_{1}(\lambda) d Z(\lambda)+\eta_{\nu}
$$

где $\lim _{\nu \rightarrow \infty} \eta_{\nu}=0$ n.6. $u$ в $L^{2}$.

2) В частности, $\lim _{\nu \rightarrow \infty} \sigma_{\nu}=\eta$ п.в. тогда и только тогда, когда

$$
\lim _{m \rightarrow \infty} \int_{0<|\lambda| \leqslant \lambda_{m}} L_{1}(\lambda) d Z(\lambda)=0 \quad \text { n.6. }
$$

Теорема 3. 1) Если выполнено условие (8) $и$

$$
\int_{0<|\lambda| \leqslant \pi} L_{1}^{2}(\lambda) L_{3}^{2}(\lambda) d F(\lambda)<\infty
$$

$m o \lim _{\nu \rightarrow \infty} \sigma_{\nu}=\eta$ n.8. $u$ в $L^{2}$.

2) Условие (13), вообще говоря, нельзя усилить. Именно, если $\omega(\lambda) \geqslant 1$,

$$
\omega(\lambda)=\omega(-\lambda), \quad \omega(\lambda)=o\left(L_{3}(\lambda)\right) \quad n p u \quad|\lambda| \rightarrow 0,
$$

то существуют унитарныи оператор $T$ и функиия $f \in L^{2}$ такие, ито

$$
\int_{0<|\lambda| \leqslant \pi} L_{1}^{2}(\lambda) \omega^{2}(\lambda) d F(\lambda)<\infty,
$$

но средние $\left(\sigma_{n}\right)$ расходятся п.в. 
Другие следствия теоремы 2 будут указаны далее. Доказательство теоремы 2 опирается на одну общую теорему, полученную в работе [2]. Сформулируем ее в нужной нам форме.

Лемма 1. Пусть $d Z_{1}(v)$ - спектральная мера с ортогональными приращениями на $0<|v| \leqslant A ;\left\|d Z_{1}(v)\right\|^{2}=d F_{1}(v) ; \int_{0<|\lambda| \leqslant A} d F_{1}(v)<$ $\infty$. Пусть $R_{n}(v)$ - последовательность непрерывных или кусочнонепрерывных функиий при $0<|v| \leqslant A$ и выполнено

У с ло в и е 1): при некоторых $\alpha>0, \beta>0, C>0$

$$
\left|R_{n}(v)\right| \leqslant C \min \left\{(n|v|)^{\alpha},(n|v|)^{-\beta}\right\} \text {. }
$$

Tогдa

$$
\lim _{m \rightarrow \infty} \int_{0<|v| \leqslant A} R_{2^{m}}(v) d Z_{1}(v)=0 \quad \text { n.в. } u \text { в } L^{2} .
$$

Пусть, кроме того, выполнено

У с ло в и е 2):

$$
\begin{aligned}
& \left|R_{n}(v)-R_{k}(v)\right| \\
& \quad \leqslant C \min \left\{|n-k|^{\alpha_{1}}|v|^{\beta_{1}} k^{-\gamma_{1}} ;|n-k|^{\alpha_{2}}|v|^{\beta_{2}} k^{-\gamma_{2}} ;(k|v|)^{-\beta_{3}}\right\}
\end{aligned}
$$

где постоянные $C>0, \alpha_{i}, \beta_{i}$ удовлетворяют условиям $\alpha_{1}>\frac{1}{2}, \alpha_{2}>\frac{1}{2}$, $\gamma_{2}>\frac{1}{2}, \beta_{3}>\frac{1}{2} ; \gamma_{1}+\beta_{1} \geqslant \alpha_{1}>\gamma_{1} ; \gamma_{2}+\beta_{2} \geqslant \alpha_{2}$.

Тогдa

$$
\lim _{n \rightarrow \infty} \int_{0<|v| \leqslant A}-R_{n}(v) d Z_{1}(v)=0 \quad \text { n.6. u } 6 L^{2} .
$$

Следуюшие несколько лемм редуцируют нашу задачу к проверке равенства (19) для ядер $R_{n}(v)$, связанных с $Q_{n}(\lambda)$. Далее буквами $C, C_{1}$ обозначаются некоторые абсолютные постоянные. В силу четности функций $Q_{n}(\lambda)$ все оценки проводятся при $0<\lambda<\pi$.

Лемма 2. Пусть $Q_{n}(\lambda)=\sum_{k=1}^{n} k^{-1} \cos k \lambda, \Phi(\lambda)=\sum_{k=1}^{\infty} k^{-1} \cos k \lambda$. При $0<\lambda \leqslant \pi, n \geqslant 2,1 \leqslant k \leqslant n$ справедливы оченки:

$$
\begin{aligned}
\left|Q_{n}(\lambda)\right| & \leqslant \ln n+C_{1} \leqslant C \log n, \\
\left|Q_{n}(\lambda)-\Phi(\lambda)\right| & \leqslant C(n \lambda)^{-1}, \\
\left|Q_{n}(\lambda)-Q_{k}(\lambda)\right| & \leqslant C \log \frac{n}{k}, \\
\left|Q_{n}(\lambda)-Q_{k}(\lambda)\right| & \leqslant C(k \lambda)^{-1} .
\end{aligned}
$$

Доказательство (20) и (22) сразу следует из неравенства $\mid Q_{n}(\lambda)-$ $Q_{k}(\lambda) \mid \leqslant \sum_{\nu=k+1}^{n} \nu^{-1}$. Далее,

$$
\begin{aligned}
\left|Q_{n}(\lambda)-\Phi(\lambda)\right| & =\left|-(n+1)^{-1} D_{n}(\lambda)+\sum_{\nu=n+1}^{\infty} \nu^{-1}(\nu+1)^{-1} D_{\nu}(\lambda)\right| \\
& \leqslant 4(n \lambda)^{-1}
\end{aligned}
$$


где $D_{n}(\lambda)$ - ядро Дирихле, $D_{n}(\lambda)=\sin \left(\left(n+\frac{1}{2}\right) \lambda\right) /(2 \sin \lambda / 2)$. Поэтому

$$
\left|Q_{n}(\lambda)-Q_{k}(\lambda)\right| \leqslant 8(k \lambda)^{-1} \quad \text { при } \quad 1 \leqslant k \leqslant n .
$$

Пусть $0<\lambda<\pi, v=(\log (2 \pi / \lambda))^{-1} ; 0<v \leqslant 1$. Обозначим $N_{m}=2^{2^{m}}$ и пусть для $2^{m} \leqslant n<2^{m+1}$

$$
R_{n}(v)=\left\{\begin{array}{lll}
v Q_{2^{n}}(\lambda) & \text { при } & 0<N_{m} \lambda \leqslant 2 \pi \\
v\left(Q_{2^{n}}(\lambda)-\Phi(\lambda)\right) & \text { при } & N_{m} \lambda>2 \pi
\end{array}\right.
$$

Положим также

$$
d Z_{1}(v)=L_{1}(\lambda) d Z(\lambda)
$$

Mера $d Z_{1}(v)$ имеет ортогональные прирашения на $(0,1]$, и условие

$$
\int_{+0}^{\pi} L_{1}^{2}(\lambda) d F(\lambda)<\infty
$$

эквивалентно условию

$$
\int_{+0}^{1} d F_{1}(v)<\infty, \quad d F_{1}(v)=\left\|d Z_{1}(v)\right\|^{2}
$$

Лемма 3. При $2^{m} \leqslant k \leqslant n \leqslant 2^{m+1}, 0<v<1$ справедливы оченки:

a)

б)

B)

$$
\begin{aligned}
\left|R_{n}(v)\right| & \leqslant C \min \left(n v ;(n v)^{-1}\right) \\
\left|R_{n}(v)-R_{k}(v)\right| & \leqslant C(n-k) v \\
\left|R_{n}(v)-R_{k}(v)\right| & \leqslant C(n-k) k^{-1}
\end{aligned}
$$

Док а з а тель с т в о. а) Если $0<N_{m} \lambda \leqslant 2 \pi$, то $n v \leqslant 2$ и $\left|R_{n}(v)\right| \leqslant v\left|Q_{2^{n}}(\lambda)\right| \leqslant C n v \leqslant 2 C \min \left(n v ;(n v)^{-1}\right) ;$ если же $N_{m} \lambda>2 \pi$, то $n v>1$, и в силу (21) имеем:

$$
\begin{aligned}
\left|R_{n}(v)\right| & \leqslant v\left|Q_{2^{n}}(\lambda)-\Phi(\lambda)\right| \leqslant C 2^{-n}\left(\lambda \log \frac{2 \pi}{\lambda}\right)^{-1} \\
& \leqslant C 2^{-m} \leqslant 2 C(n v)^{-1}
\end{aligned}
$$

Поэтому $\left|R_{n}(v)\right| \leqslant 2 C \min \left(n v ;(n v)^{-1}\right)$ и в области $N_{m} \lambda>2 \pi$.

Аналогично, при $2^{m} \leqslant k \leqslant n \leqslant 2^{m+1}$ из (22) следует неравенство б):

$$
\left|R_{n}(v)-R_{k}(v)\right| \leqslant v\left|Q_{2^{n}}(\lambda)-Q_{2^{k}}(\lambda)\right| \leqslant C(n-k) v .
$$

Если $k v \geqslant 2$, то б) $\Longrightarrow$ в); если же $n v>k v>2$, то $N_{m} \lambda \geqslant 2 \pi$ и оценка в) тривиально следует из (27): $\left|R_{n}(v)-R_{k}(v)\right| \leqslant 4 C k^{-1} \leqslant C_{1}(n-k) k^{-1}$.

Лемма 4. Если $\left(a_{k}\right)-$ произвольные числа,

$$
S_{n}=\sum_{1}^{n} a_{k} \quad u \quad \lim _{\nu \rightarrow \infty} \nu^{-1} S_{\nu}=0
$$


mo $u$

$$
\lim _{n \rightarrow \infty} \sup _{1 \leqslant k \leqslant 2^{n}}\left|\sum_{\nu=2^{n}+1}^{2^{n}+k} \nu^{-1} a_{\nu}\right|=0 .
$$

Действительно,

$$
\begin{aligned}
\left|\sum_{\nu=2^{n}+1}^{2^{n}+k} \nu^{-1} a_{\nu}\right| & =\left|-\left(2^{n}+1\right)^{-1} S_{2^{n}}+\sum_{\nu=2^{n}+1}^{2^{n}+k} \nu^{-1}(\nu+1)^{-1} S_{\nu}\right| \\
& \leqslant 2^{-n}\left|S_{2^{n}}\right|+\sup _{2^{n}<\nu \leqslant 2^{n+1}} \nu^{-1}\left|S_{\nu}\right|
\end{aligned}
$$

Лемма 5. Если выполнено условие (9), то

$$
\begin{aligned}
2 \int_{0<|\lambda| \leqslant \lambda_{m}} \Phi(\lambda) d Z(\lambda) & =2 \int_{0<|\lambda| \leqslant \lambda_{m}} L_{1}(\lambda) d Z(\lambda)+\delta_{m}, \\
\lim _{m \rightarrow \infty} \delta_{m} & =0 \quad \text { n.в. } u \text { в } L^{2} .
\end{aligned}
$$

Д о к а з а т е л в с т в о. При $0<|\lambda| \leqslant \pi$ известна оценка (см. [11, гл. 5]): $\Phi(\lambda)=L_{1}(\lambda)+\Psi_{1}(\lambda),\left|\Psi_{1}(\lambda)\right| \leqslant C_{1}$. Если обозначить

$$
\delta_{m}=2 \int_{0<|\lambda| \leqslant \lambda_{m}} \Psi_{1}(\lambda) d Z(\lambda)
$$

то из условия (9) легко следует сходимость ряда $\sum 2^{2^{m}}\left\|\delta_{m}\right\|^{2}$, что влечет за собой (29).

Д ок а 3 а т ел ь т в о те о ре м ы 2. Пусть выполнены условия (8)-(10). Оценим сначала разность $\sigma_{2^{n}}-\eta$, где $2^{m} \leqslant n<2^{m+1}$. Полагая $\lambda_{m}=2 \pi N_{m}^{-1}$, имеем:

$$
\begin{aligned}
\sigma_{2^{n}}-\eta= & 2 \int_{0<|\lambda| \leqslant \pi}\left[Q_{2^{n}}(\lambda)-\Phi(\lambda)\right] d Z(\lambda) \\
= & -2 \int_{0<|\lambda| \leqslant \lambda_{m}} \Phi(\lambda) d Z(\lambda)+2 \int_{0<|\lambda| \leqslant \lambda_{m}} Q_{2^{n}}(\lambda) d Z(\lambda) \\
& +2 \int_{\lambda_{m}<|\lambda| \leqslant \pi}\left[Q_{2^{n}}(\lambda)-\Phi(\lambda)\right] d Z(\lambda)
\end{aligned}
$$

Таким образом,

$$
\sigma_{2^{n}}=\eta-2 \int_{0<|\lambda| \leqslant \lambda_{m}} \Phi(\lambda) d Z(\lambda)+\varepsilon_{n},
$$

где остаток $\varepsilon_{n}$ представляется в виде суммы двух интегралов $\varepsilon_{n}^{\prime}$ и $\varepsilon_{n}^{\prime \prime}$ по промежуткам $0<\lambda \leqslant \pi$ и $-\pi \leqslant \lambda<0$. Первый интеграл переписывается в виде

$$
\varepsilon_{n}^{\prime}=\int_{+0}^{1} R_{n}(v) d Z_{1}(v), \quad \int_{+0}^{1} d F_{1}(v)<\infty
$$


В силу четности ядра $Q_{n}(\lambda)$ второй интеграл переписывается в виде

$$
\varepsilon_{n}^{\prime \prime}=\int_{+0}^{1} R_{n}(v) d Z_{2}(v), \quad \int_{+0}^{1} d F_{2}(v)<\infty
$$

где ядра $R_{n}(\lambda)$ определены согласно (24). Оценки а), б), в) леммы 3 показывают, что для ядер $R_{n}(\lambda)$ выполнены все требования леммы 1 (при $\alpha=\beta=\alpha_{1}=\alpha_{2}=\gamma_{2}=\beta_{1}=\beta_{3}=1 ; \gamma_{1}=0, \beta_{2}=0$ ). Значит,

$$
\lim _{n \rightarrow \infty} \varepsilon_{n}=0 \quad \text { п.в. и в } L^{2} \text {. }
$$

Для завершения доказательства применяем леммы 4 и 5 . Тогда при $N_{m} \leqslant \nu<N_{m+1}$ получим требуемое представление (11), где для $\nu=2^{n}+k, 2^{m} \leqslant n<2^{m+1}, 1 \leqslant k<2^{n}$, введены обозначения:

$$
\eta_{\nu}=-\delta_{m}+\varepsilon_{n}+\left(\sigma_{\nu}-\sigma_{2^{n}}\right), \quad\left|\eta_{\nu}\right| \leqslant\left|\delta_{m}\right|+\left|\varepsilon_{n}\right|+\sup _{1 \leqslant k<2^{n}}\left|\sigma_{2^{n}+k}-\sigma_{2^{n}}\right|
$$

3 а м е ч а н и е. Вместо последовательности $N_{m}=2^{2^{m}}$ в теореме 2 можно рассматривать любую последовательность $\widetilde{N}_{m}=\left[q_{1}^{q_{2}^{m}}\right], q_{1}>1$, $q_{2}>1$, или любую другую последовательность $\tilde{N}_{m}$, для которой $\log \log \widetilde{N}_{m}=A m+O(1) ; \tilde{\lambda}_{m}=\widetilde{N}_{m}^{-1}$. Тогда число решений неравенств $N_{m} \leqslant \widetilde{N}_{n}<N_{m+1}$ и $\widetilde{N}_{m} \leqslant \widetilde{N}_{m}<\widetilde{N}_{m+1}$ не превосходит $C=C(A)$ при $m=1,2, \ldots$ и условие (12) эквивалентно условию

$$
\lim _{m \rightarrow \infty} \int_{0<|\lambda| \leqslant \tilde{\lambda}_{m}} L_{1}(\lambda) d Z(\lambda)=0 \quad \text { п.в. }
$$

Д ок а за т е л ь с т в т т о р е м ы 3. С помощью представления (11) сведем задачу к задаче о сходимости п.в. некоторого ассоциированного ортогонального ряда $\sum_{1}^{\infty} \Phi_{k}$ (аналогично [1], [2]). Если $\Phi_{k}=$ $2 \int_{\lambda_{k+1}<|\lambda| \leqslant \lambda_{k}} L_{1}(\lambda) d Z(\lambda)$, то $\left(\Phi_{k}, \Phi_{n}\right)=0$ при $k \neq n$, и в силу (9) имеем: $\sum_{1}^{\infty} \Phi_{k} \stackrel{L^{2}}{=} \eta, \sum\left\|\Phi_{k}\right\|^{2}<\infty$. Так как $\log k \leqslant L_{3}(\lambda) \leqslant \log (k+1)$ при $\lambda_{k}<|\lambda| \leqslant \lambda_{k+1}$, то по условио (13) $\sum\left\|\Phi_{k}\right\|^{2} \log ^{2} k<\infty$. По теореме Меньшова-Радемахера последовательность

$$
\sum_{k=m}^{\infty} \Phi_{k}=\eta-\sum_{k=1}^{m-1} \Phi_{k}
$$

сходится п.в. к 0, т.е. выполнено условие (12). Вторая часть теоремы получается с помошью известного контрпримера Меньшова (ср. [1], [2]).

Пусть $N_{\nu}=2^{2^{\nu}}$ и $\lambda_{\nu}=2 \pi N_{\nu}^{-1}$. Если $\omega(u)=o(\log \log \log u)$ при $u \rightarrow \infty$, то для $\omega_{1}(\nu)=\omega\left(\lambda_{\nu}\right)$ имеем: $\omega_{1}(\nu) \geqslant 1, \omega_{1}(\nu)=o(\log \nu)$ при $\nu \rightarrow \infty$. Поэтому в $L^{2}[0,1]$ существует такая ортогональная система $\left\{\Psi_{\nu}(x)\right\}_{1}^{\infty}$, что $\sum\left\|\Psi_{\nu}\right\|^{2} \omega_{1}^{2}(\nu)<\infty$, но ряд $\sum_{\nu=1}^{\infty} \Psi_{\nu}(x)$ сходится в $L^{2}$ и расходится п.в. (см. [12]). Рассмотрим замкнутую линейную оболочку 
системы $\left\{\Psi_{\nu}\right\}_{1}^{\infty}=H_{1}$ и в ее ортогональном дополнении $H_{2}$ выберем ортонормированный базис $\left\{\Phi_{\mu}\right\}$.

Введем унитарный оператор $T$ в $L^{2}[0,1]$ формулами:

$$
T \Psi_{\nu}=\exp \left(i \lambda_{\nu}\right) \Psi_{\nu} \quad(\nu \geqslant 1) ; \quad T \Phi_{\mu}=\Phi_{\mu} .
$$

Для элемента $f(x) \stackrel{L^{2}}{=} \sum_{\nu=1}^{\infty} \Psi_{\nu}(x) 2^{-\nu}$ имеем:

$$
T^{k} f(x)=\sum_{1}^{\infty} \exp \left(i \lambda_{\nu} k\right) \Psi_{\nu}(x) 2^{-\nu}
$$

Поэтому $T f=\int_{-\pi}^{\pi} e^{i \lambda} d Z(\lambda)$, где спектральная мера $d Z(\lambda)$ имеет скачки $\Psi_{\nu}(x) 2^{-\nu}$ в точках $\lambda_{\nu}(\nu \geqslant 1)$. Так как $L_{1}\left(\lambda_{\nu}\right)=2^{\nu}$, то по теореме 2

$$
\sigma_{N_{m}}(x)=\sum_{k=1}^{N_{m}} k^{-1}\left(T^{k} f(x)+T^{-k} f(x)\right)=\eta(x)-2 \sum_{\nu=m}^{\infty} \Psi_{\nu}(x)+\eta_{N_{m}}(x) .
$$

Поэтому средние $\sigma_{N_{m}}$ расходятся п.в. С другой стороны,

$$
\int_{0<|\lambda| \leqslant \pi} L_{1}^{2}(\lambda) \omega^{2}(\lambda) d F(\lambda)=\sum\left\|\Psi_{\nu}\right\|^{2} \omega_{1}^{2}(\nu)<\infty
$$

3 а м е ч а н и е. Представление (32) для данного оператора $T$ и функции $f$ легко проверить и непосредственным подсчетом.

Приведем также достаточные условия существования одностороннего преобразования Гильберта, выраженные через «корреляционную функцию» $R_{1}(n)=\left(T^{k} f, T^{k+n} f\right)$ или «усредненную коррелящионную функцию»

$$
R_{2}(n)=n^{-2}\left\|\sum_{1}^{n} T^{k} f\right\|^{2}=n^{-1}\|f\|^{2}+2 n^{-2} \sum_{k=1}^{n}(n-k) R_{1}(k) .
$$

Известно, что определенные ограничения на спектральную меру $d F(\lambda)$ при $\lambda \rightarrow 0$ переписываются в виде соответствуюших условий, налагаемых на скорость убывания $R_{2}(n)$. В частности, условие (13) (вместе с условием (8)) эквивалентно сходимости ряда

$$
\sum R_{2}(n) n^{-1} \log n \log \log \log ^{2} n
$$

(см., например, [13]). Кроме того, сходимость ряда

$$
\sum R_{1}(n) n^{-1} \log n \log \log \log ^{2} n
$$

влечет за собой сходимость ряда (33). Отсюда получается следующая теорема. 
Теорема 3а. Если сходится ряд (33) или ряд (34), то

$$
\lim _{k \rightarrow \infty} \sum_{k=1}^{n} k^{-1} T^{k} f(x)=\eta^{\prime \prime}(x) \quad \text { n.6. }
$$

В работе [13] теорема 3а доказана другим, более сложным, методом. Там же содержится пример, показывающий неулучшаемость, в определенном смысле, условий теоремы 3 а.

Отметим одну интересную задачу, возникаюшую в связи с данным кругом вопросов. Известно, что для сохраняюших меру унитарных преобразований пространства с конечной мерой в себя и порожденных ими унитарных операторов двустороннее эргодическое преобразование Гильберта всегда сходится п.в. и в $L^{2}$ (для $f \in L^{2}$ ). В работах [14], [15] было показано, что это утверждение можно вывести из цитированного выше результата работы [3] (см. соотношение (6)). Более того, для таких операторов не только при $\varepsilon_{m}=2^{-m}$, но и при любых $\varepsilon_{m} \rightarrow+0$ справедливы равенства

$$
\lim _{m \rightarrow \infty} \int_{0<\lambda \leqslant \varepsilon_{m}} d Z(\lambda)=0, \quad \lim _{m \rightarrow \infty} \int_{-\varepsilon_{m} \leqslant \lambda<0} d Z(\lambda)=0 \quad \text { п.в. }
$$

Возникает вопрос: всегда ли из сходимости в $L^{2}$ односторонних средних $\sum_{k=1}^{n} k^{-1} f\left(U^{k} x\right)$, где $f \in L^{2}, U-$ сохраняющее меру преобразование $\Omega$ в себя, следует сходимость этих средних п.в.? Для решения этого вопроса было бы достаточно установить, что условие (9) в этом случае влечет за собой сходимость п.в. (при любых $\varepsilon_{k} \rightarrow+0$ или при $\varepsilon_{k}=2 \pi 2^{-2^{k}}$ ) последовательности интегралов $\left\{\int_{0<\lambda \leqslant \varepsilon_{k}} L_{1}(\lambda) d Z(\lambda)\right\}$. Если $\left(\xi_{k}\right)$ - гауссовская стационарная последовательность, $\xi_{k}=\xi_{0}\left(U^{k} x\right)$, то $Z(\lambda)$ - гауссовский процесс с независимыми прирацениями и ответ на поставленный вопрос положительный.

\section{§2. Обобшенные эргодические преобразования}

Рассмотрим задачу о сходимости в $L^{2}$ и почти всюду более обших двусторонних и односторонних эргодических преобразований

$$
\sum_{k=-n}^{n} a_{k} T^{k} f(x), \quad \sum_{k=-n}^{n} a_{k} \operatorname{sign} k T^{k} f(x), \quad \sum_{k=1}^{n} a_{k} T^{k} f(x),
$$

где $a_{-k}=a_{k}, a_{0}=0$ и $\left(a_{k}\right)_{1}^{\infty}$ - некоторая стремящаяся к нулю последовательность. В этом параграфе исследуется случай $a_{k}=k^{-\alpha}, \alpha>0$, в $\S 3$ приводятся некоторые обобщения. Пусть снова $T-$ унитарный оператор, $f \in L^{2}$,

$$
\tilde{\sigma}_{n}^{\prime}=\sum_{k=1}^{n} k^{-\alpha}\left(T^{k} f(x)-T^{-k} f(x)\right) ; \quad \tilde{\sigma}_{n}^{\prime \prime}=\sum_{k=1}^{n} k^{-\alpha} T^{k} f(x) ;
$$




$$
\tilde{\sigma}_{n}=\sum_{k=1}^{n} k^{-\alpha}\left(T^{k} f(x)+T^{-k} f(x)\right)
$$

Используя спектральное представление (2), получим, что

$$
\begin{aligned}
& \widetilde{\sigma}_{n}^{\prime}=2 i \int_{|\lambda| \leqslant \pi} S_{n}^{\alpha}(\lambda) d Z(\lambda) ; \quad \tilde{\sigma}_{n}=2 \int_{|\lambda| \leqslant \pi} C_{n}^{\alpha}(\lambda) d Z(\lambda), \\
& \widetilde{\sigma}_{n}^{\prime \prime}=\frac{1}{2}\left(\tilde{\sigma}_{n}^{\prime}+\tilde{\sigma}_{n}\right)
\end{aligned}
$$

где

$$
\begin{aligned}
& C_{n}^{\alpha}(\lambda)=\sum_{k=1}^{n} k^{-\alpha} \cos k \lambda \\
& S_{n}^{\alpha}(\lambda)=\sum_{k=1}^{n} k^{-\alpha} \sin k \lambda .
\end{aligned}
$$

Таким образом, достаточно исследовать сходимость интегралов (35) при $n \rightarrow \infty$. Так как случай $\alpha>1$ тривиален (для любой $f \in L^{2}$ средние $\widetilde{\sigma}_{n}, \widetilde{\sigma}_{n}^{\prime}, \widetilde{\sigma}_{n}^{\prime \prime}$ сходятся абсолютно п.в.), то далее считаем, что $0<\alpha<1$.

Известно, что при $\lambda \neq 0$

$$
\begin{array}{llll}
\lim _{n \rightarrow \infty} C_{n}^{\alpha}(\lambda)=c_{\alpha}(\lambda), & c_{\alpha}(\lambda) \sim A_{\alpha}|\lambda|^{\alpha-1} & \text { при } & \lambda \rightarrow 0, \\
\lim _{n \rightarrow \infty} S_{n}^{\alpha}(\lambda)=s_{\alpha}(\lambda), & s_{\alpha}(\lambda) \sim B_{\alpha}|\lambda|^{\alpha-1} & \text { при } & \lambda \rightarrow 0,
\end{array}
$$

где $A_{\alpha}=\Gamma(1-\alpha) \sin (\pi \alpha / 2), B_{\alpha}=\Gamma(1-\alpha) \cos (\pi \alpha / 2)($ см. [11]). При этом

$$
\lim _{n \rightarrow \infty} C_{n}^{\alpha}(0)=\infty ; \quad \lim _{n \rightarrow \infty} S_{n}^{\alpha}(0)=0 .
$$

Из этих замечаний получаем следуюшее утверждение.

Теорема 4. Пусть $0<\alpha<1$. Средние $\widetilde{\sigma}_{n}^{\prime}$ сходятся в $L^{2}$ mогда $u$ только тогда, когда выполнено условие

$$
\int_{0<|\lambda| \leqslant \pi}|\lambda|^{2 \alpha-2} d F(\lambda)<\infty
$$

nричем $\lim _{n \rightarrow \infty} \widetilde{\sigma}_{n}^{\prime} \stackrel{L^{2}}{=} 2 i \int_{0<|\lambda| \leqslant \pi} s_{\alpha}(\lambda) d Z(\lambda)=\eta_{\alpha}$.

Средние $\widetilde{\sigma}_{n}$ и $\widetilde{\sigma}_{n}^{\prime \prime}$ сходятся в $L^{2}$ mогда и только тогда, когда вьполнено условие (42) и условие (8). При этом

$$
\lim _{n \rightarrow \infty} \tilde{\sigma}_{n}^{\prime} \stackrel{L^{2}}{=} 2 \int_{0<|\lambda| \leqslant \pi} c_{\alpha}(\lambda) d Z(\lambda)=\xi_{\alpha} ; \quad \lim _{n \rightarrow \infty} \tilde{\sigma}_{n}^{\prime \prime} \stackrel{L^{2}}{=} \frac{1}{2}\left(\xi_{\alpha}+\eta_{\alpha}\right) .
$$

Теорема 5. Пусть выполнено условие (42). 1) Если $0<\alpha<1$, то справедливо представление

$$
\tilde{\sigma}_{2^{m}}^{\prime}=\eta_{\alpha}-2 i B_{\alpha} \int_{0<|\lambda| \leqslant 2^{-m}}|\lambda|^{\alpha-1} \operatorname{sign} \lambda d Z(\lambda)+\gamma_{2^{m}}
$$




$$
\lim _{m \rightarrow \infty} \gamma_{2^{m}}=0 \quad \text { n.6. } u \text { в } L^{2} .
$$

В иастности, средние $\widetilde{\sigma}_{2^{m}}^{\prime}$ сходятся п.в. и в $L^{2}$ тогда и только mozдa, когдa

$$
\lim _{m \rightarrow \infty} \int_{0<|\lambda| \leqslant 2^{-m}}|\lambda|^{\alpha-1} \operatorname{sign} \lambda d Z(\lambda)=0 \quad \text { n.e. }
$$

2) Если $\frac{1}{2}<\alpha<1$, то также и при $2^{m} \leqslant n<2^{m+1}$ справедливо представление

$$
\begin{gathered}
\tilde{\sigma}_{n}^{\prime}=\eta_{\alpha}-2 i B_{\alpha} \int_{0<|\lambda| \leqslant 2^{-m}}|\lambda|^{\alpha-1} \operatorname{sign} \lambda d Z(\lambda)+\gamma_{n}, \\
\lim _{n \rightarrow \infty} \gamma_{n}=0 \quad \text { n.6. } u \text { в } L^{2} .
\end{gathered}
$$

В частности, средние $\widetilde{\sigma}_{n}^{\prime}$ сходятся п.в. и в $L^{2}$ тогда и только тогда, когда выполнено условие (45).

3 а м е ч а н и е. Аналогичная теорема имеет место и для средних $\widetilde{\sigma}_{n}^{\prime \prime}, \widetilde{\sigma}_{n}$ со следуюшими отличиями: а) кроме условия (42) необходимо предполагать также, что выполнено условие (8); б) вместо представления $\left(43^{\prime}\right)$ или (43) рассматривается представление с четной подынтегральной функцией, например, при $\alpha>\frac{1}{2}, 2^{m} \leqslant n<2^{m+1}$.

$$
\begin{gathered}
\widetilde{\sigma}_{n}=\xi_{\alpha}-2 A_{\alpha} \int_{0<|\lambda| \leqslant 2^{-m}}|\lambda|^{\alpha-1} d Z(\lambda)+\delta_{n}, \\
\lim _{n \rightarrow \infty} \delta_{n}=0 \quad \text { п.в. и в } L^{2} ;
\end{gathered}
$$

в) представление средних $\widetilde{\sigma}_{n}^{\prime \prime}$ получается как линейная комбинация представлений $\widetilde{\sigma}_{n}$ и $\widetilde{\sigma}_{n}^{\prime}$.

Доказательство теоремы 5 проводится по той же схеме, что и доказательство теоремы 2 (и несколько проще). Будем оценивать, для определенности, интегралы $\int_{0<|\lambda| \leqslant \pi} S_{n}^{\alpha}(\lambda) d Z(\lambda)$. Рассмотрим при $2^{m} \leqslant$ $n<2^{m+1}$ ядра

$$
\widetilde{S}_{n}^{\alpha}(\lambda)=\left\{\begin{array}{lll}
S_{n}^{\alpha}(\lambda)|\lambda|^{1-\alpha} & \text { при } & |\lambda| \leqslant 2^{-m} \\
\left(S_{n}^{\alpha}(\lambda)-s_{\alpha}(\lambda)\right)|\lambda|^{1-\alpha} & \text { при } & |\lambda|>2^{-m}
\end{array}\right.
$$

Лемма 6. Справедливы оченки:

a) $\left.\quad\left|\widetilde{S}_{n}^{\alpha}(\lambda)\right| \leqslant C_{\alpha} \min \left((n|\lambda|)^{1-\alpha},(n|\lambda|)\right)^{-\alpha}\right)$;

б) $\left|\widetilde{S}_{n}^{\alpha}(\lambda)-\widetilde{S}_{k}^{\alpha}(\lambda)\right| \leqslant C_{\alpha}(n-k)|\lambda|^{1-\alpha} k^{-\alpha} \quad\left(2^{m} \leqslant k<n \leqslant 2^{m+1}\right)$;

в) $\left|\widetilde{S}_{n}^{\alpha}(\lambda)-\widetilde{S}_{k}^{\alpha}(\lambda)\right| \leqslant C_{\alpha}(k|\lambda|)^{-\alpha} \quad\left(2^{m} \leqslant k<n \leqslant 2^{m+1}\right)$.

До к а 3 а т е льс т в о. Если $2^{m}|\lambda| \leqslant 1$, то $n|\lambda| \leqslant 2$, и

$$
\left|\widetilde{S}_{n}^{\alpha}(\lambda)\right| \leqslant|\lambda|^{1-\alpha} \sum_{1}^{n} \nu^{-\alpha} \leqslant C_{\alpha}(n|\lambda|)^{1-\alpha} .
$$


Если же $2^{m}|\lambda|>1$, то и $n|\lambda|>1$; преобразование Абеля дает:

$$
\begin{aligned}
\left|\widetilde{S}_{n}^{\alpha}(\lambda)\right| & =\left|\sum_{k=n+1}^{\infty} k^{-\alpha} \sin k \lambda\right| \cdot|\lambda|^{1-\alpha} \\
& \leqslant\left|-(n+1)^{-\alpha} D_{n}(\lambda)+\sum_{k=n+1}^{\infty} D_{k}(\lambda) \Delta\left(k^{-\alpha}\right)\right| \cdot|\lambda|^{1-\alpha} \\
& \leqslant \bar{C}_{\alpha}(n|\lambda|)^{-\alpha} .
\end{aligned}
$$

Оценка в) вытекает из а). Наконец, при всех $\lambda$ и $k<n$

$$
\left|\widetilde{S}_{n}^{\alpha}(\lambda)-\widetilde{S}_{k}^{\alpha}(\lambda)\right| \leqslant|\lambda|^{1-\alpha} \sum_{\nu=k+1}^{n} \nu^{-\alpha} \leqslant(n-k) k^{-\alpha}|\lambda|^{1-\alpha} .
$$

Лемма 7. Если выполнено условие (42) при некотором $\alpha>0$, то

$$
\begin{aligned}
& 2 i \int_{0<|\lambda| \leqslant 2-m} s_{\alpha}(\lambda) d Z(\lambda) \\
& =-2 i B_{\alpha} \int_{0<|\lambda| \leqslant 2^{-m}}|\lambda|^{1-\alpha} \operatorname{sign} \lambda d Z(\lambda)+\delta_{m}^{\alpha}, \\
& \lim _{m \rightarrow \infty} \delta_{m}^{\alpha}=0 \quad \text { n. в. } u \in L^{2} .
\end{aligned}
$$

Эта лемма доказывается так же, как лемма 5.

Доказате льст в о те о ремы 5 . При $2^{m} \leqslant n<2^{m+1}$

$$
\begin{aligned}
\tilde{\sigma}_{n}^{\prime}-\eta_{\alpha} & =2 i \int_{0<|\lambda| \leqslant \pi}\left[S_{n}^{\alpha}(\lambda)-s_{k}^{\alpha}(\lambda)\right] d Z(\lambda) \\
& =-2 i \int_{0<|\lambda| \leqslant 2^{-m}} s_{\alpha}(\lambda) d Z(\lambda)+\eta_{n}^{\alpha}
\end{aligned}
$$

где

$$
\begin{gathered}
\eta_{n}^{\alpha}=2 i \int_{0<|\lambda| \leqslant \pi} \widetilde{S}_{n}^{\alpha}(\lambda) d Z_{1}(\lambda), \\
d Z_{1}(\lambda)=|\lambda|^{\alpha-1} d Z(\lambda), \quad d F_{1}(\lambda)=\left\|d Z_{1}(\lambda)\right\|^{2} .
\end{gathered}
$$

Спектральная мера $d Z_{1}(\lambda)$ также имеет ортогональные приращения, причем $\int_{0<|\lambda| \leqslant \pi} d F_{1}(\lambda)<\infty$ в силу условия (42). Применим теперь лемму 1 к величинам $\eta_{n}^{\alpha}$. Если $0<\alpha<1$, то по лемме 6 для ядер $\widetilde{S}_{n}^{\alpha}(\lambda)$ выполнено условие 1 ) леммы 1 , и поэтому $\lim _{m \rightarrow \infty} \eta_{2^{m}}^{\alpha}=0$ п.в. и в $L^{2}$. Используя также лемму 7 , получим первое утверждение теоремы 5. Если $\alpha>\frac{1}{2}$, то применимо и условие 2) леммы 1 (в силу оценок леммы 6 можно взять $\alpha_{1}=\alpha_{2}=1, \beta_{1}=\beta_{2}=1-\alpha, \gamma_{1}=\gamma_{2}=\beta_{3}=\alpha>\frac{1}{2}$, и выполнены условия $\left.\beta_{1}+\gamma_{1}=1>\gamma_{1}, \beta_{2}+\gamma_{2}=1 \geqslant \alpha_{2}\right)$. Поэтому справедливо и второе утверждение теоремы 5 . 
Из теоремы Меньшова-Радемахера и представлений (43), (43') сразу вытекает следующее предложение (его аналоги справедливы и для $\left.\widetilde{\sigma}_{n}, \widetilde{\sigma}_{n}^{\prime \prime}\right)$.

Теорема 6. 1) Пусть выполнено условие

$$
\int_{0<|\lambda| \leqslant \pi}|\lambda|^{2 \alpha-2} L_{2}(\lambda) d F(\lambda)<\infty
$$

Eсли $1>\alpha>0$, mo $\lim _{m \rightarrow \infty} \tilde{\sigma}_{2^{m}}^{\prime}=\eta_{\alpha}$ n.e.; еслu $1>\alpha>\frac{1}{2}$, mo $u$ $\lim _{n \rightarrow \infty} \tilde{\sigma}_{n}^{\prime}=\eta_{\alpha}$ n.8.

2) Условие (50) является неулучшаемым в том же смысле, ито и условие (13) в теореме 3 .

Аналогом теоремы 3а является следующее утверждение.

Теорема 6а. Пусть сходится ряд

$$
\sum R_{2}(n) n^{1-2 \alpha} \log \log ^{2} n
$$

или сxодится ряд

$$
\sum R_{1}(n) n^{1-2 \alpha} \log \log ^{2} n .
$$

Если $0<\alpha<1$, то средние $\widetilde{\sigma}_{2^{m}}, \widetilde{\sigma}_{2^{m}}^{\prime}, \widetilde{\sigma}_{2^{m}}^{\prime \prime}$ сходятся п.в. и в $L^{2} ;$ если $\frac{1}{2}<\alpha<1$, mо и средние $\widetilde{\sigma}_{n}, \widetilde{\sigma}_{n}^{\prime}, \tilde{\sigma}_{n}^{\prime \prime}$ cxodrmca n.в. и в $L^{2}$.

Действительно, сходимость второго ряда влечет за собой сходимость первого ряда, которая, в свою очередь, влечет сходимость интеграла (50) и условие (8) (см. [13]).

Утверждение теоремы 6а при $\alpha>\frac{1}{2}$ с помошью более сложных прямых оценок было получено в работе [13].

3 а м е ч а н и е. При $0<\alpha \leqslant \frac{1}{2}$ не только теряют силу утверждение 2) теоремы 5 (и соответствующие утверждения теорем 6 и $6 \mathrm{a}$ ), но и другие, более сильные, чем (42), ограничения на спектральную меру не влекут за собой сходимость средних $\widetilde{\sigma}_{n}\left(\widetilde{\sigma}_{n}^{\prime}, \widetilde{\sigma}_{n}^{\prime \prime}\right)$ почти всюду.

II р и м е р. Пусть $\left(\chi_{n}\right)_{0}^{\infty}$ - полная ортонормированная система Хаара в $L^{2}[0,1]$. Она обладает следуюшими свойствами:

$$
\varlimsup_{n \rightarrow \infty} n^{-1 / 2}\left|\chi_{n}\right| \geqslant \frac{1}{2} \quad \text { п.в.; } \varliminf_{n \rightarrow \infty} n^{-1 / 2}\left|\chi_{n}\right|=0 \quad \text { п.в. }
$$

Положим $\varphi_{0}=\chi_{0}, \varphi_{-1}=\chi_{1}, \varphi_{1}=\chi_{2}, \varphi_{2}=\chi_{3}, \ldots$ и образуем стащионарный процесс

$$
\xi_{n}=\varphi_{n}-\varphi_{n+1}=U^{n}\left(\varphi_{0}-\varphi_{1}\right), \quad-\infty<n<\infty,
$$

$U$ - унитарный оператор сдвига. Рассмотрим, например, односторонние средние

$$
\tilde{\sigma}_{n}^{\prime \prime}=\sum_{k=1}^{n} k^{-\alpha} \xi_{k}=-n^{-\alpha} \varphi_{n+1}+\sum_{k=1}^{n} \varphi_{k} b_{k}^{\alpha} .
$$

Здесь $b_{k}^{\alpha}=O\left(k^{-\alpha-1}\right)$, и поэтому ряп $\sum_{1}^{\infty} \varphi_{k} b_{k}^{\alpha}$ абсолютно сходится п.в. 
Следовательно, предел $\left(\tilde{\sigma}_{n}^{\prime \prime}\right)$ существует тогда и только тогда, когда существует предел последовательности $\left(n^{-\alpha} \varphi_{n+1}\right)$. В силу (51) при $\alpha \leqslant \frac{1}{2}$ последний предел не существует п.в. В то же время

$$
\begin{aligned}
& d F(\lambda)=\left|1-e^{i \lambda}\right|^{2} d \lambda=4 \sin ^{2} \frac{\lambda}{2} d \lambda \\
& R_{2}(n)=O\left(n^{-2}\right) ; \quad R_{1}(n)=0 \quad(n \geqslant 2) .
\end{aligned}
$$

Следовательно,

$$
\begin{array}{rrr}
\int_{0<|\lambda| \leqslant \pi}|\lambda|^{-\beta} d F(\lambda)<\infty & \text { при } & \beta<3 \\
\sum R_{2}(n) n^{\gamma}<\infty & \text { при } & \gamma<1 .
\end{array}
$$

\section{§. Некоторые обобщения}

3.1. Преобразования с общими весовыми коэффициентами. Пусть $b(\tau)-$ положительная медленно меняющаяся. функция (м.м.ф.) на $[1, \infty)$, т.е. $\forall \delta>0 \quad b(\tau) \tau^{-\delta} \downarrow 0, b(\tau) \tau^{\delta} \uparrow \infty$ при $\tau \rightarrow \infty$.

Вместо средних $\widetilde{\sigma}_{n}, \widetilde{\sigma}_{n}^{\prime}, \widetilde{\sigma}_{n}^{\prime \prime}$ из $\S 2$ можно рассмотреть средние с коэффициентами $a_{k}=b(k) k^{-\alpha}$. Приведем формулировки для средних $\tilde{\sigma}_{n}^{\prime}$ $\left(a_{-k}=a_{k} \operatorname{sign} k\right)$; для $\tilde{\sigma}_{n}$ и $\tilde{\sigma}_{n}^{\prime \prime}$ они аналогичны.

Пусть

$$
\tilde{\sigma}_{n}^{\prime}=\sum_{k=1}^{n} b(k) k^{-\alpha}\left[T^{k} f(x)-T^{-k} f(x)\right], \quad f \in L^{2}
$$

Тогда $\widetilde{\sigma}_{n}^{\prime}=2 i \int_{|\lambda| \leqslant \pi} \widetilde{S}_{n}^{\alpha}(\lambda) d Z(\lambda)$,

$$
\widetilde{S}_{n}^{\alpha}(\lambda)=\sum_{k=1}^{n} b(k) k^{-\alpha} \sin k \lambda ; \quad \widetilde{s}_{\alpha}(\lambda)=\sum_{k=1}^{\infty} b(k) k^{-\alpha} \sin k \lambda
$$

Пусть сначала $0<\alpha<1$.

Теорема 4'. Средние $\tilde{\sigma}_{n}^{\prime}$ сходятся в $L^{2}$ тогда и только тогда, когдa

$$
\int_{0<|\lambda| \leqslant \pi}|\lambda|^{2 \alpha-2} b^{2}\left(\frac{1}{|\lambda|}\right) d F(\lambda)<\infty
$$

причем $\lim _{n \rightarrow \infty} \widetilde{\sigma}_{n} \stackrel{L^{2}}{=} 2 i \int_{0<|\lambda| \leqslant \pi} \widetilde{s}_{\alpha}(\lambda) d Z(\lambda)=\widetilde{\eta}_{\alpha}$.

Теорема 5'. Пусть выполнено условие (52). 1) Если $0<\alpha<1$, то

$$
\begin{gathered}
\widetilde{\sigma}_{2^{m}}^{\prime}=\widetilde{\eta}_{\alpha}-2 i B_{\alpha} \int_{0<|\lambda| \leqslant 2^{-m}}|\lambda|^{\alpha-1} \operatorname{sign} \lambda b\left(\frac{1}{|\lambda|}\right) d Z(\lambda)+\gamma_{2^{m}}, \\
\lim _{m \rightarrow \infty} \gamma_{2^{m}}=0 \quad \text { n.в. } u \text { в } L^{2}
\end{gathered}
$$


В частности, средние $\widetilde{\sigma}_{2 m}^{\prime}$ сходятся в п.в. и в $L^{2}$ тогда и только moгдa, когдa

$$
\lim _{m \rightarrow \infty} \int_{0<|\lambda| \leqslant 2^{-m}}|\lambda|^{\alpha-1} \operatorname{sign} \lambda b\left(\frac{1}{|\lambda|}\right) d Z(\lambda)=0 \quad \text { n.e. }
$$

2) Если $\frac{1}{2}<\alpha<1$, то такэе и при всех $2^{m} \leqslant n<2^{m+1}$

$$
\begin{gathered}
\widetilde{\sigma}_{n}^{\prime}=\widetilde{\eta}_{\alpha}-2 i B_{\alpha} \int_{0<|\lambda| \leqslant 2^{-m}}|\lambda|^{\alpha-1} \operatorname{sign} \lambda b\left(\frac{1}{|\lambda|}\right) d Z(\lambda)+\gamma_{n}, \\
\lim _{n \rightarrow \infty} \gamma_{n}=0 \quad \text { n.в. u } \theta L^{2} .
\end{gathered}
$$

$B$ частности, $\lim _{n \rightarrow \infty} \tilde{\sigma}_{n}^{\prime}=0$ n.в. и в $L^{2}$ тогда и только тогда, когда выполнено условие (52).

Доказательства этих утверждений проводятся подобно доказательству теоремы 5 с использованием известных свойств соответствующих ядер (см. $[11$, гл. 5]). Более интересен случай $\alpha=1$. Пусть, по-прежнему, $b(\tau)$ - некоторая м.м.Ф. на $[1, \infty), b(-\tau)=b(\tau)$. Приведем соответствуюшие результаты для средних

$$
\begin{aligned}
& \sigma_{n}^{\prime}=\sum_{k=1}^{n} b(k) k^{-1}\left[T^{k} f(x)-T^{-k} f(x)\right], \\
& \sigma_{n}=\sum_{k=1}^{n} b(k) k^{-1}\left[T^{k} f(x)+T^{-k} f(x)\right] .
\end{aligned}
$$

Как и при $b(k) \equiv 1$, свойства этих средних существенно отличаются друг от друга. Обозначим

$$
\begin{array}{ll}
Q_{n}^{\prime}(\lambda)=\sum_{k=1}^{n} b(k) k^{-1} \sin k \lambda ; & \Psi(\lambda)=\sum_{k=1}^{\infty} b(k) k^{-1} \sin k \lambda ; \\
Q_{n}(\lambda)=\sum_{k=1}^{n} b(k) k^{-1} \cos k \lambda ; & \Phi(\lambda)=\sum_{k=1}^{\infty} b(k) k^{-1} \cos k \lambda .
\end{array}
$$

Заметим, что если интеграл $\int_{1}^{\infty} b(\tau) \tau^{-1} d \tau$ сходится, то ряды $\sum_{1}^{\infty} b(k) \times$ $k^{-1} T^{k} f, \sum_{1}^{\infty} b(k) k^{-1} T^{-k} f$ абсолютно сходятся п.в. и в $L^{2}$ для любых $f \in L^{2}$. Поэтому далее считаем, что

$$
B(t)=\int_{1}^{t} b(\tau) \tau^{-1} d \tau, \quad \lim _{t \rightarrow \infty} B(t)=\infty .
$$

Лемма 8. $П р и|\lambda| \rightarrow 0$ имеем:

$$
\Psi(\lambda) \sim \frac{\pi}{2} b\left(\frac{1}{|\lambda|}\right), \quad \Phi(\lambda) \sim B\left(\frac{1}{|\lambda|}\right) \sim B\left(\frac{2 \pi}{|\lambda|}\right)
$$

(cм. [11]). 
Образуем ядра $R_{n}(\lambda)$ для $2^{m} \leqslant n<2^{m+1}$ :

$$
R_{n}(\lambda)=\left\{\begin{array}{lll}
Q_{n}^{\prime}(\lambda) b^{-1}\left(\frac{1}{|\lambda|}\right) & \text { при } & 0<|\lambda| \leqslant 2^{-m} \\
\left(Q_{n}^{\prime}(\lambda)-\Psi(\lambda)\right) b^{-1}\left(\frac{1}{|\lambda|}\right) & \text { при } & |\lambda|>2^{-m}
\end{array}\right.
$$

С использованием известной асимптотики $Q_{n}^{\prime}(\lambda)$ легко проверяются все условия јеммы 3 для этих ядер, и получается представление средних $\sigma_{n}^{\prime}$ и его естественные следствия. Приведем лишь одно из них.

Теорема 7. Для сходимости средних $\sigma_{n}^{\prime}$ n.в. $u$ в $L^{2}$ nрu $n \rightarrow \infty$ необходимо и достаточно, чтобы

$$
\lim _{m \rightarrow \infty} \int_{0<|\lambda| \leqslant 2^{-m}} b\left(\frac{1}{|\lambda|}\right) \operatorname{sign} \lambda d Z(\lambda)=0 \quad \text { n.в. u } 6 L^{2} .
$$

В частности, если

$$
\int_{0<|\lambda| \leqslant 2^{-m}} b^{2}\left(\frac{1}{|\lambda|}\right) \log \log ^{2}\left(\frac{4 \pi}{|\lambda|}\right) d F(\lambda)<\infty,
$$

то средние $\sigma_{n}^{\prime}$ сходятся п.в. и в $L^{2}$ nрu $n \rightarrow \infty$.

Следствие. $Е с л и ~ b(k)=O(\log \log k)^{-1} n p u k \rightarrow \infty$, mо для любого унитарного оператора.T и яюбой функции $f \in L^{2}$ существует п.в. обобщенное эргодическое преобразование Гильберта

$$
\lim _{n \rightarrow \infty} \sum_{k=1}^{n} b(k) k^{-1}\left[T^{k} f(x)-T^{-k} f(x)\right]
$$

Для средних $\sigma_{n}$ ситуация иная. Если выполнено условие (54), то необходимы дополнительные условия на спектральную меру $d Z(\lambda)$ при $\lambda \rightarrow 0$, чтобы обеспечить сходимость п.в. и в $L^{2}$ средних $\sigma_{n}$.

Укажем нужные изменения в формулировках и доказательствах теорем 1-3 в этом случае. Для функции $B(t)$, определенной согласно (54), введем последовательности действительных чисел $1<N_{1}^{\prime}<N_{2}^{\prime}<\cdots$ и $N_{1}<N_{2}<\cdots: B\left(N_{n}^{\prime}\right)=n ; N_{m}=N_{2^{m}}^{\prime}(m \geqslant 1)$.

Обозначим $Q_{n}(\lambda)=\sum_{k=1}^{n} b(k) k^{-1} \cos k \lambda$. Пусть $v=[B(2 \pi / \lambda)]^{-1}$ при $0<\lambda \leqslant \pi ;$ тогда $0<v \leqslant v_{0}=[B(2)]^{-1}$. Для $2^{m} \leqslant n<2^{m+1}$; $0<v \leqslant v_{0}$ положим

$$
R_{n}(v)= \begin{cases}v Q_{N_{n}^{\prime}}(\lambda) & \text { при } 0<N_{m} \lambda \leqslant 2 \pi, \\ v\left(Q_{N_{n}^{\prime}}(\lambda)-\Phi(\lambda)\right) & \text { при } N_{m} \lambda>2 \pi\end{cases}
$$

Используя известную асимптотику сумм $Q_{n}(\lambda)$ и $\Phi(\lambda)-Q_{n}(\lambda)$, получаем, что длй ядер $R_{n}(v)$ справедлива лемма 3 . Отсюда следуют приводимые ниже аналоги теорем $1-3$. 
Теорема 1'. Средние $\sigma_{n}$ сходятся в $L^{2}$ тогда и только тогда, когда выполнено условие (8) $u$

$$
\int_{0<|\lambda| \leqslant \pi} B^{2}\left(\frac{2 \pi}{|\lambda|}\right) d F(\lambda)<\infty
$$

Теорема 2'. Пусть выполнены условия (8) $u$ (55), и пусть $B\left(N_{m}\right)=2^{m}$. Тогда при $N_{m} \leqslant n<N_{m+1}, \lambda_{m}=2 \pi N_{m}^{-1}$ справедливо представление (11) с заменой функиии $\log (2 \pi /|\lambda|)$ на функиию $B(2 \pi /|\lambda|)$. $B$ частности, $\lim _{n \rightarrow \infty} \sigma_{n}=\eta$ n.в. и в $L^{2}$ тогда и только тогда, когда

$$
\lim _{m \rightarrow \infty} \int_{0<|\lambda| \leqslant \lambda_{m}} B\left(\frac{2 \pi}{|\lambda|}\right) d Z(\lambda)=0 \quad \text { n.в. }
$$

Пусть $B_{1}(\lambda)=\log m$ при $\lambda_{m+1}<|\lambda| \leqslant \lambda_{m}, m \geqslant 1$.

Теорема $3^{\prime}$. Если выполнено условие.

$$
\int_{0<|\lambda| \leqslant \pi} B^{2}\left(\frac{2 \pi}{|\lambda|}\right) B_{1}^{2}(\lambda) d H(\lambda)<\infty,
$$

mо средние $\sigma_{n}$ сходатся п.в. и в $L^{2}$ и условие (56) является в определенном смысле неусиляемым.

3 а м е ч а н и е. Вместо последовательности $N_{m}$ в теореме $2^{\prime}$ можно взять любую последовательность $\bar{N}_{m}$, для которой $\log B\left(\bar{N}_{m}\right)=$ $A m+O(1) ; A>0$.

Следствие. Средние

$$
\sum_{k=1}^{n} \frac{T^{k} f(x)}{k \ln (k+1)}
$$

cxoдgmca n.в. $и$ в $L^{2}$, ecлu

$$
\int_{0<|\lambda| \leqslant \pi} L_{2}^{2}(\lambda) L_{4}^{2}(\lambda) d F(\lambda)<\infty
$$

( и бункцию $L_{4}(\lambda)$ нельзя, вообще говоря, заменить другой, медленкее растущей функцией).

Для доказательства в теореме 3 полагаем $b(u)=[\ln (u+1)]^{-1}$, $B(t)=\ln \ln (t+1)$ и берем $N_{m}=e^{e^{2^{m}}}, B_{1}(\lambda) \sim c L_{4}(\lambda)$ при $\lambda \rightarrow 0$.

3.2. Эргодические преобразования для других классов операторов и случайных пропессов. Все полученные выше результаты опираются, в основном, на спектральное представление оператора $T$. Поскольку спектральным представлением обладают и некоторые другие классы операторов и случайных процессов (причем не только в пространстве $L^{2}$, но и в других $L^{p}$ ), в работах [4]-[9] и др. эргодические теоремы и эргодические преобразования обобщались в различных направлениях. При доказательстве этих результатов основную роль играет 
общая теорема о сходимости последовательности стохастических интегралов, доказанная в работе [2] и относяшаяся не только к спектральным мерам с ортогональными приращениями, но и к более общим спектральным мерам.

Кратко укажем ряд возможных обобщений результатов этой работы.

1. Эргодические преобразования для нормальных операторов. Если $T$ - нормальный оператор в $L^{2},\|T\| \leqslant 1, f \in L^{2}$, то

$$
T f=\int_{|\zeta| \leqslant 1} \zeta \cdot Z(d \zeta) ; \quad F(d \zeta)=\|Z(d \zeta)\|^{2} ; \quad\|T f\|^{2}=\int_{|\zeta| \leqslant 1} F(d \zeta) .
$$

Индивидуальная эргодическая теорема в терминах спектральной меры $Z(d \zeta)$, определенной на борелевских подмножествах единичного круга, была получена в [16]. Для средних

$$
\sigma_{n}^{\prime \prime}=\sum_{k=1}^{n} k^{-1} T^{k} f(x)=\int_{|\zeta| \leqslant 1}\left(\sum_{k=1}^{n} k^{-1} \zeta^{k}\right) Z(d \zeta)
$$

можно получить аналоги теорем 1-3. Обозначим $N_{m}=2^{2^{m}}$,

$$
K=\{\zeta \in \mathbf{C}:|\zeta| \leqslant 1, \zeta \neq 1\}, \quad D_{m}=K \cap\left\{\zeta:|1-\zeta| \leqslant N_{m}^{-1}\right\} .
$$

Пусть $\ln (1 /(1-\zeta))$ - главная ветвь соответствующей аналитической функции, $\ln (1 /(1-\zeta))=\sum_{k=1}^{\infty} k^{-1} \zeta^{k}(\zeta \in K)$.

Теорема 8. Пусть $f \in L^{2}$. Ecsu $F(\{1\})=0$,

$$
\int_{K}\left|\ln \frac{1}{1-\zeta}\right|^{2} F(d \zeta)<\infty \quad u \quad \eta \stackrel{L^{2}}{=} \int_{K} \ln \frac{1}{1-\zeta} Z(d \zeta)
$$

то справедливо представление: $п р и N_{m} \leqslant n<N_{m+1}$

$$
\sigma_{n}=\eta-\int_{D_{m}} \ln \frac{1}{1-\zeta} Z(d \zeta)+\eta_{n}, \quad \lim _{n \rightarrow \infty} \eta_{n}=0 \quad \text { n.в. } u \text { в } L^{2} \text {. }
$$

Для средних $\sum_{1}^{n} b(k) k^{-\alpha} T^{k} f$, где $b(k)$ - м.м.ф., $0<\alpha \leqslant 1$, а $T-$ нормальный оператор, также справедливы аналоги теорем 4-6.

2. Непрерывные аналоги. Рассмотрим группу унитарных операторов $\left\{T^{t},-\infty<t<\infty\right\}$ в $L^{2}(\Omega)$, обладаюшую спектральным представлением $T^{t} f=\xi(t)=\int_{\mathbf{R}} e^{i \lambda t} d Z(\lambda) ; f \in L^{2}$.

Если $b(\tau)$ - м.м.ф., $0<\alpha \leqslant 1$, то для интегралов

$$
\sigma_{t}=\int_{1}^{t} b(\tau) \tau^{-\alpha} \xi(\tau) d \tau ; \quad \sigma_{t}^{\prime}=\int_{1}^{t} b(\tau) \tau^{-\alpha}[\xi(\tau)-\xi(-\tau)] d \tau
$$

при $t \rightarrow \infty$ справедливы аналоги предыдущих теорем.

Отметим, что подобным же образом изучается сходимость п.в. при $\varepsilon \rightarrow+0$ интегралов

$$
\widehat{\sigma}_{\varepsilon}=\int_{1}^{\varepsilon} b(\tau) \tau^{-\alpha} \xi(\tau) d \tau ; \quad \hat{\sigma}_{\varepsilon}^{\prime}=\int_{1}^{\varepsilon} b(\tau) \tau^{-\alpha}[\xi(\tau)-\xi(-\tau)] d \tau
$$


Как и в локальной эргодической теореме (относящейся к сходимости п.в. средних $\varepsilon^{-1} \int_{0}^{\varepsilon} \xi(\tau) d \tau$ при $\varepsilon \rightarrow+0$, см. [17]), необходимые и достаточные условия выражаются через свойства спектральной меры на множествах $\left\{\lambda:|\lambda| \geqslant \lambda_{m}\right\}$.

3. Гармонизуемые случайные прочессы. В теории случайных процессов наряду со стационарными процессами рассматриваются различные классы нестационарных, но гармонизуемых процессов. Например, процесс называется сильно гармонизуемым (или гармонизуемым в смысле Лозва), если справедливо представление $\xi(t)=\int_{\mathbf{R}} e^{i \lambda t} Z(d \lambda)$, где $\mathbf{E}(Z(d \lambda) \cdot \overline{Z(d \mu)})=F(d \lambda, d \mu)-$ конечная мера на $\mathbf{R} \times \mathbf{R}$. Усиленный закон больших чисел для этих процессов был получен в работах [2], [4]; в работе [6] он распространен на более широкий класс слабо гармонизуемых процессов. Для всех этих случаев справедливы аналоги теорем $1,2,4,5$. Например, средние $\sigma_{T}$ сильно гармонизуемого процесса $\xi(t)$ имеют вид

$$
\sigma_{T}=\int_{1}^{T} \tau^{-1}(\xi(\tau)+\xi(-\tau)) d \tau
$$

Из спектрального представления следует, что $\sigma_{T}=2 \int_{\mathbf{R}} \widetilde{Q}_{T}(\lambda) Z(d \lambda)$, где

$$
\widetilde{Q}_{T}(\lambda)=\int_{\lambda}^{T \lambda} \frac{\cos u}{u} d u ; \quad \lim _{T \rightarrow \infty} \widetilde{Q}_{T}(\lambda)=-c i(\lambda)=\int_{\lambda}^{\infty} \frac{\cos u}{u} d u ;
$$

$-c i(\lambda) \sim \ln (1 /|\lambda|)$ при $\lambda \rightarrow 0 ;|c i(\lambda)| \leqslant C$ при $|\lambda| \geqslant 1$.

Обозначим $D=\left\{(\lambda, \mu): 0<\lambda^{2}+\mu^{2} \leqslant 1\right\}$. Если выполнены условия $F(\{0,0\})=0$ и

$$
\iint_{D} \ln \frac{1}{|\lambda|} \ln \frac{1}{\mu} F(d \lambda, d \mu)<\infty,
$$

то средние $\sigma_{T}$ сходятся в $L^{2}$ при $T \rightarrow \infty$ к $\eta \in L^{2}, \eta=-2 \int_{\mathbf{R}} c i(\lambda) Z(d \lambda)$.

Далее, если $N_{m}=2^{2^{m}}, \lambda_{m}=N_{m}^{-1}$, то при $N_{m} \leqslant T<N_{m+1}$ справедливо представление

$$
\sigma_{T}=\eta-2 \int_{0<|\lambda| \leqslant \lambda_{m}} \ln \frac{1}{|\lambda|} Z(d \lambda)+\eta_{T} ; \quad \lim _{T \rightarrow \infty} \eta_{T}=0 \quad \text { п.в. и в } L^{2} .
$$

В частности, сходимость п.в. при $T \rightarrow \infty$ средних $\sigma_{T}$ эквивалентна условию

$$
\lim _{m \rightarrow \infty} \int_{0<|\lambda| \leqslant \lambda_{m}} \ln \frac{1}{|\lambda|} Z(d \lambda)=0 \quad \text { п.в. }
$$

Интересно, что многие из этих результатов обобщаются и на операторы, действующие в пространствах $L^{p}(1<p<\infty)$. Так, в работе [9] получен усиленный закон больших чисел для так называемых $(\alpha, \beta)$ ограниченных процессов в терминах стохастических спектральных мер этих процессов. В работах [8], [18] показано, что ограниченные и обратимые операторы $T: L^{p} \rightarrow L^{p}$, для которых $\sup _{n \in \mathbf{Z}}\left\|T^{n}\right\|<\infty$, также имеют 
некоторое спектральное представление. В работах [9], [18] для средних $n^{-1} \sum_{k=1}^{n} T^{k} f$ и $\sum_{k=1}^{n} k^{-1}\left[T^{k} f-T^{-k} f\right]$ получены спектральные критерии сходимости почти всюду. На эти случаи можно распространить и ряд результатов данной статьи.

\section{СПИСОК ЛИТЕРАТУРЫ}

1. Гапашжия B. Ф. Критерии усиленного закона больших чисел для классов стационарных в широком смысле процессов и однородных случайных полей. - Теория вероятн. и ее примен., 1977, т. XXII, в. 2, с. 295-319.

2. Гапошкия В. Ф. Одна теорема о сходимости почти всюду последовательности измеримых функций и ее применения к последовательностям стохастических интегралов. - Матем. сб., 1977, т. 104, в. 1, с. 3-21.

3. Jajte $R$. On the existence of the ergodic Hilbert transform. - Ann. Probab., 1987, v. 15, № 2, p. 831-835.

4. Rousseau-Egelé J. La loi forté des grands nombres pour les processus harmonisables. - Ann. Inst. H. Poincaré, 1979, v. 15, p. 175-186.

5. Cambanis S., Hardin $C$., Weron A. Ergodic properties of certain stationary stable processes. - Stoch. Process. Appl., 1987, v. 24, № 1, p. 1-18.

6. Dehay D. Strong law of large numbers for weakly harmonizable processes. - Stoch. Process. Appl., 1987, v. 24, № 2, p. 259-267.

7. Bradley R. E. Pseudospectral operators and the pointwise ergodic theorem. - Proc. Amer. Math. Soc., 1991, v. 112, p. 863-870.

8. Houdré $C$. Harmonizability, $V$-boundedness, $(2, p)$-boundedness of stochastic processes. - Probab. Theory Relat. Fields, 1990, v. 84, p. 39-54.

9. Houdré $C$. On spectral SLLN and pointwise ergodic theorem in $L^{\alpha}$. - Preprint, Dept. of Math. Univ. of Maryland, 1991.

10. Клесов О. И. О сходимости почти всюду кратных рядов Котельникова-Шеннона. - Проблемы передачи информации, 1984, т. 20, с. 79-93.

11. Зигмунд А. Тригонометрические ряды. Т. І. М.: Мир, 1965, 615 с.

12. Качмаж C., IIтейжгауз $Г$. Теория ортогональных рядов. М.: Физматгиз, 1958, $507 \mathrm{c}$.

13. Гапошкия В.Ф. Сходимость рядов, связанных со стационарными последовательностями. - Изв. АН СССР, сер. матем., 1975, т. 39, в. 6, с. 1366-1392.

14. Campbell J., Petersen $K$. The spectral measure and Hilbert transform of a measurepreserving transformation. - Trans. Amer. Math. Soc., 1989, v. 313, p. 121-129.

15. Assani $I$., Petersen $K$. The helical transform as a connection between ergodic theory and harmonic analysis. - Trans. Amer. Math. Soc., 1992, v. 331, p. 131-142.

16. Гапошкия $B$. $Ф$. Об индивидуальной эргодической теореме для нормальных операторов в $L^{2}$. - Функцион. анализ и его прилож., 1981, т. 15, № 1, с. 131-142.

17. Гапоикин $B$. $\Phi$. Локальная эргодическая теорема для групп унитарных операторов и стационарных процессов второго порядка. - Матем. с6., 1980, т. 111, c. $249-261$.

18. Berkson E., Bourgain J., Gillespie T. A. On the a.e. convergence of ergodic averages for power-bounded operators on $L^{p}$-spaces. - Integral Equations and Operator Theory, 1991, v. 14, p. 678-715.

Поступила в редакцию 16.VI.1993 